\title{
Combination of treatment with death receptor 5-specific antibody with therapeutic HPV DNA vaccination generates enhanced therapeutic antitumor effects
}

\author{
Chih-Wen Tseng ${ }^{1,6}$, Cornelia Trimble ${ }^{1,2,4}$, Archana Monie ${ }^{1}$, Ronald D. Alvarez ${ }^{7}$, Warner K. \\ Huh $^{7}$, Donald J. Buchsbaum ${ }^{8}$, J. Michael Straughn Jr. ${ }^{7}$, Mei-Cheng Wang ${ }^{5}$, Hideo Yagita ${ }^{9}$, \\ Chien-Fu Hung ${ }^{1,4}$, and T.-C. Wu ${ }^{1,2,3,4}$ \\ ${ }^{1}$ Department of Pathology, Johns Hopkins Medical Institutions, Baltimore, Maryland, USA \\ ${ }^{2}$ Department of Obstetrics and Gynecology, Johns Hopkins Medical Institutions, Baltimore, \\ Maryland, USA \\ ${ }^{3}$ Department of Molecular Microbiology and Immunology, Johns Hopkins Medical Institutions, \\ Baltimore, Maryland, USA \\ ${ }^{4}$ Department of Oncology, Johns Hopkins Medical Institutions, Baltimore, Maryland, USA \\ ${ }^{5}$ Department of Biostatistics, Johns Hopkins Medical Institutions, Baltimore, Maryland, USA \\ ${ }^{6}$ Department of Obstetrics \& Gynecology, Chang Gung Memorial Hospital, Kaohsiung Medical \\ Center, Chang Gung University College of Medicine, Taiwan \\ ${ }^{7}$ Department of Obstetrics and Gynecology, University of Alabama at Birmingham, Birmingham, \\ Alabama 35249, USA \\ ${ }^{8}$ Department of Radiation Oncology, University of Alabama at Birmingham, Birmingham, Alabama \\ 35249, USA \\ ${ }^{9}$ Department of Immunology, Juntendo University School of Medicine, Tokyo, Japan
}

\begin{abstract}
There is currently a vital need for the development of novel therapeutic strategies for the control of advanced stage cancers. Antigen-specific immunotherapy and the employment of antibodies against the death receptor 5 (DR5) have emerged as two potentially promising strategies for cancer treatment. In the current study, we hypothesize that the combination of treatment with the anti-DR5 monoclonal antibody, MD5-1 with a DNA vaccine encoding calreticulin (CRT) linked to human papillomavirus type 16 (HPV-16) E7 antigen (CRT/E7(detox)) administered via gene gun would lead to further enhancement of E7-specific immune responses as well as antitumor effects. Our results indicated that mice bearing the E7-expressing tumor, TC-1 treated with MD5-1 monoclonal antibody followed by CRT/E7(detox) DNA vaccination generated the most potent therapeutic anti-tumor effects as well as highest levels of E7-specific CD8+ T cells among all the groups tested. In addition, treatment with MD5-1 monoclonal antibody was capable of rendering the TC-1 tumor cells more susceptible to lysis by E7-specific cytotoxic T lymphocytes. Our findings serve as an important foundation for future clinical translation.
\end{abstract}

Address correspondence to Dr. T.-C. Wu, Department of Pathology, The Johns Hopkins University School of Medicine, CRB II Room 309, 1550 Orleans Street, Baltimore, MD 21231, USA. Phone: (410) 614-3899; Fax: (443) 287-4295; E-Mail: wutc@jhmi.edu. 


\section{Keywords}

anti-DR5 antibody; DNA vaccine; calreticulin (CRT); E7; human papillomavirus (HPV)

\section{Introduction}

Conventional therapies including chemotherapy, surgery and radiation are not very effective at controlling advanced stage cancers. Thus, there is a need for the development of novel therapies for the treatment of these advanced stage cancers. Antigen-specific immunotherapy using DNA vaccines and employment of a monoclonal antibody $(\mathrm{mAb})$ against the death receptor 5 (DR5) expressed on tumor cells represent two innovative therapies that may potentially be developed and employed in conjunction with conventional therapies.

Antigen-specific immunotherapy using DNA vaccines has become a promising approach due to its safety, stability and ease of preparation (for review, see [1,2]). However, DNA vaccines suffer from low immunogenicity because they do not have the intrinsic ability to amplify and spread like some viral vectors. Thus, in order to overcome this limitation of DNA vaccines, many strategies have been developed to enhance the DNA vaccine potency, such as directly targeting the DNA into dendritic cells (DCs) in vivo intradermally via gene gun as well as the modification of the properties of antigen-presenting cells (APCs) (for review see [3-5]).

We have previously developed several intracellular targeting strategies to improve DNA vaccine potency by enhancing MHC class I/II antigen presentation and processing in DCs in preclinical models. One particular strategy explores the linkage of calreticulin (CRT), a $\mathrm{Ca}^{2+}$ binding protein located in the endoplasmic reticulum (ER) (for review, see [6]) to a model tumor antigen, human papilloma virus type-16 (HPV-16) E7, for the development of a DNA vaccine, CRT/E7 [7]. Among the various intracellular targeting strategies tested, we observed that mice vaccinated intradermally with DNA encoding CRT/E7 generated one of the greatest enhancement in E7-specific CD8+ T cell immune responses and antitumor effects against an E7-expressing tumor, TC-1 [8]. The encouraging preclinical data has prompted us to propose a phase I clinical trial in HPV-16+ patients with stage IB1 cervical cancer using the pNGVL4aCRT/E7(detox) formulated in the gene gun device suitable for clinical usage. The pNGVL4a vector was obtained from the NIH National Gene Vector Laboratory and has previously been used in clinical trials [9]. The E7(detox) represents the HPV-16 E7 gene that has been mutated at the Rb binding site of E7, abolishing the capacity of E7 to transform cells [10-12]. Thus, the current clinical-grade DNA construct represents a potentially useful reagent for the control of HPV-16-associated lesions.

Another innovative approach for the control of cancer is the employment of mAbs that are capable of leading to apoptotic tumor cell death. For example, binding of $\mathrm{mAb}$ to tumor necrosis factor (TNF)-related apoptosis-inducing ligand (TRAIL) DR5 via its extracellular domain has been shown to initiate apoptosis via its cytoplasmic domain by activation of downstream caspases in the extrinsic apoptotic pathway [13-17]. We have previously demonstrated the antitumor effects of a mAb against the DR5 receptor in several tumor models [15,18-20]. In addition, it has been found that the binding of antibodies to DR5 would lead to apoptotic tumor cell death [21]. Furthermore, we have shown that several human cancers, including cervical cancers express DR5 on their surface [15]. In fact, a fully human anti-DR5 mAb (lexatumumab) is now in phase I clinical trials in patients with advanced solid malignancies [22].

In the current study, we hypothesize that the combination of treatment with the anti-DR5 mAb, MD5-1 with DNA vaccination with pNGVL4a-CRT/E7(detox) administered via gene gun may lead to significant enhancement of E7-specific CD8+ T cell immune responses as well as 
antitumor effects. We have previously demonstrated that agents that are capable of causing apoptotic tumor cell death can effectively be used in combination with E7 DNA vaccination to generate enhanced E7-specific CD8+ T cell immune responses and antitumor effects [23, 24]. Our results indicated that E7-expressing TC-1 tumors expressed DR5. Furthermore, TC-1 tumor-bearing mice treated with MD5-1 mAb followed by CRT/E7(detox) DNA vaccination generated significantly higher frequency of E7-specific CD8+ T cells and better therapeutic anti-tumor effects compared treatment with MD5-1 mAb alone or DNA vaccination alone. In addition, treatment with MD5-1 mAb rendered the TC-1 tumor cells more susceptible to lysis by E7-specific CTLs. Our findings serve as an important foundation for future clinical translation.

\section{Materials and Methods}

\section{Mice}

Six- to 8-week-old female C57BL/6 mice were purchased from the National Cancer Institute (Frederick, MD) and housed in the oncology animal facility of Johns Hopkins Hospital (Baltimore, MD). All animal procedures were performed according to approved protocols and in accordance with recommendations for the proper use and care of laboratory animals.

\section{Cell lines and antibodies}

We previously generated an E7-expressing tumorigenic cell line, TC-1 [25] and a firefly luciferase-expressing TC-1 cell line (TC-1-luc). The anti-DR5 mAb, MD5-1 was purified from ascites using a $\mathrm{G}$ protein column as described previously [26]. The phycoerythrin (PE) labeled MD5-1 mAb was obtained from eBioscience, San Diego, CA.

\section{DNA constructs and gene gun vaccination}

The pNGVL4a vector was obtained from the NIH National Gene Vector Laboratory. A secondgeneration plasmid from pNGVL-3, pNGVL4a encodes a kanamycin-resistance gene and a transcription unit consisting of a CMV promoter, a multiple cloning site, and a poly-A tail. It contains two short immunostimulatory DNA sequences (ISS) in the non-coding region of the backbone. The ISS sequences are derived from a bacterial ampicillin-resistance gene and consist of tandem repeats of a CpG dinucleotide in the base context, 5'-AACGTT-3'. It has been demonstrated that an ISS-containing pDNA can elicit the production of IFN- $\gamma$ and IL-12 in transfected keratocytes and dermal APCs in mice, which results in a potent $\mathrm{T}$ helper cell type 1 response. This vector also contains a transcription unit that yields high levels of antigen expression and its DNA adjuvant unit elicits strong immunologic Th1 type responses against the pDNA-encoded protein. E7 oncogenic protein detox form has substituted amino acids at positions 24 (glycine in place of cysteine) and 26 (glycine in place of glutamic acid). These substitutions have been shown to inhibit E7 protein binding to phospho retinoblastoma (pRb) at position 11, abating E7's ability to transform cells. Thus, it more adequately used in clinical trials.

For the gene gun-mediated intradermal vaccination, DNA-coated gold particles ( $1 \mu \mathrm{g}$ DNA/ bullet) were delivered to the shaved abdominal region of C57BL/6 mice using a helium-driven gene gun (BioRad, Hercules, CA, USA) with a discharge pressure of 400 p.s.i., according to manufacturer protocol. C57BL/6 mice were vaccinated via gene gun with 2 bullets of 4a-CRT/ E7(detox) for a total of $2 \mu \mathrm{g}$ DNA. These mice received two booster vaccinations of the same regimen at 4-day intervals. 


\section{In vivo treatment experiments}

C57BL/6 mice were inoculated subcutaneously with $5 \times 10^{4} /$ mouse of TC- 1 cells on day 0 . The tumor-bearing mice were divided into four groups ( 5 per group) based on treatment regimen: control (no treatment), MD5-1, 4a-CRT/E7(detox), or MD5-1 and 4aCRT/E7(detox). For the administration of MD5-1, $100 \mu \mathrm{l}$ of a $2.5 \mathrm{mg} / \mathrm{ml}$ solution of MD5-1 was injected intraperitoneally on day 8 after tumor inoculation. For the gene-gun administration of $4 \mathrm{a}-\mathrm{CRT} /$ E7(detox) DNA, $2 \mu \mathrm{g}$ was injected into the tumor-bearing mice on days 11, 15, and 19 after tumor inoculation.

\section{Intracellular cytokine staining by flow cytometry for immune assays}

Immune assays were done in different immune response models. The mice were inoculated with $5 \times 10^{4} \mathrm{TC}-1$ tumor cells and treated with the same treatments as described in the above in vivo treatment experiments. Splenocytes were harvested from different groups of mice 7 days after the last vaccination with 4a-CRT/E7d DNA. Prior to intracellular cytokine staining, $5 \times 10^{6} /$ mouse of pooled splenocytes from each vaccination group were incubated for $16 \mathrm{~h}$ with $1 \mu \mathrm{g} / \mathrm{ml}$ of E7 peptide containing a H2-D ${ }^{\mathrm{b}}$-restricted CTL epitope (aa 49-57) for detecting antigen-specific CD8 ${ }^{+}$T-cell precursors in the presence of GolgiPlug (BD Pharmingen, San Diego, CA). Intracellular IFN- $\gamma$ staining and flow cytometry analysis were performed as described previously [27]. Analysis was performed on a Becton-Dickinson FACScan with CELLQuest software (Becton Dickinson Immunocytometry System, Mountain View, CA, USA).

\section{Cytotoxic assay}

TC-1-luc cells with or without MD5-1 coating plated in the amount of $1 \times 10^{5} /$ well in a 24well plate served as the target cells. For MD5-1- coated TC-1-luc cells, $5 \mu \mathrm{lof} 2.5 \mathrm{mg} / \mathrm{ml}$ solution of MD5-1 in $0.5 \mathrm{ml}$ of FACS buffer was added to TC-1-luc cells and incubated for $30 \mathrm{~min}$ at $4^{\circ} \mathrm{C}$. The mixture was then washed with $3 \mathrm{ml} \mathrm{FACS}$ buffer solution and resuspended in CTL medium. MD5-1-coated TC-1-luc cells were counted and seeded into wells ( 2 lanes) next to the non-coated TC-1-luc cells (2 lanes) and incubated overnight $(18 \mathrm{hrs})$ at $37^{\circ} \mathrm{C}$. The total number of effector cells (CTLs) was assigned according to an 11:1 (E:T) ratio. CTLs were added in the amount of $1 \times 10^{5}$ /well to both types of target cells (coated and non-coated) and the effector-target cell mixture was incubated for $4.5 \mathrm{hrs}$. Luciferin $\left(1.3 \times 10^{-3} \mathrm{mg}\right.$ per well) then was added to the wells for optical imaging with Xenogen IVIS 200.

\section{Tumor measurement and conditional survival}

Three-dimensional tumor sizes were monitored beginning on day 6 after tumor challenge at 3day intervals by measurement with a dial caliper. Tumor sizes were approximated by measuring the longest (length) and shortest dimension (width). Tumor volume was calculated by the following formula: tumor volume $=0.52 \times$ length $\times$ width $^{2}$. At the end of the experiment, the mice were euthanized when tumor size reached $>20 \mathrm{~mm}$ in major diameter.

\section{Statistical analysis}

All data are expressed as means \pm S.E. and are representative of at least two independent experiments. Comparisons between individual data points were made by Student $t$ test. KaplanMeier survival curves for tumor treatment experiments were applied. To determine the significance of differences between curves, $p$ values were calculated using a log-rank test. $p$ 0.05 was considered significant. 


\section{Results}

\section{E7-expressing TC-1 tumors express the death receptor 5}

In order to determine if the E7-expressing TC-1 tumor is a suitable model for determining the effect of MD5-1 mAb in the treatment of tumor-bearing mice, we characterized the expression of DR5 using flow cytometry analysis with the MD5-1 antibody on TC-1 tumor cells. TC-1 cells were stained with PE-conjugated MD5-1 mAb to detect the expression of DR5. An isotype-matched control antibody was used as a negative control. As shown in Figure 1, significant expression of DR5 were observed in the TC-1 tumor cells stained with the MD5-1 antibody (green profile) compared to TC-1 cells stained with the isotype control (purple profile). Thus, our data indicate that TC-1 tumor cells demonstrate surface expression of DR5 and may serve as a potentially suitable model for testing the therapeutic effect of MD5-1 on tumor-bearing mice.

\section{TC-1 tumor-bearing mice treated with MD5-1 mAb followed by CRT/E7(detox) DNA generate the best therapeutic anti-tumor effects}

To determine the therapeutic antitumor effects generated by MD5-1 mAb combined with vaccination with DNA encoding CRT/E7(detox), we first challenged groups of C57BL/6 mice (5 per group) with TC-1 tumor cells and then treated them with MD5-1 mAb and/or the DNA vaccine as illustrated in Figure 2A. As shown in Figure 2B, tumor-bearing mice treated with MD5-1 mAb followed by CRT/E7(detox) DNA showed significantly reduced tumor volumes over time as compared to tumor-bearing mice treated with MD5-1 alone or the DNA vaccine alone $(p<0.05)$. Furthermore, tumor-bearing mice treated with MD5-1 mAb followed by CRT/ E7(detox) DNA showed improved survival compared to tumor-bearing mice treated with MD5-1 alone or the DNA vaccine alone $(p=0.01)$ (Figure 3). Thus, our data indicate that the treatment regimen using MD5-1 mAb followed by CRT/E7(detox) DNA produces the best therapeutic anti-tumor effects and long-term survival in TC-1 tumor-bearing mice.

\section{TC-1 tumor-bearing mice treated with MD5-1 mAb followed by CRT/E7(detox) DNA generate highest frequency of E7-specific CD8+ T cells}

In order to determine the E7-specific CD8+ $\mathrm{T}$ cell immune response in tumor-bearing mice treated with MD5-1 and the CRT/E7(detox) DNA vaccine, we first challenged groups of C57BL/6 mice (5 per group) with TC-1 tumor cells and then treated them with DNA vaccine alone, MD5-1 mAb alone, or MD5-1 mAb followed by DNA vaccination as illustrated in Figure 2A. Seven days after the last treatment, we harvested splenocytes from vaccinated mice and characterized the presence of $\mathrm{E} 7$-specific $\mathrm{CD} 8^{+} \mathrm{T}$ cells in treated mice using intracellular cytokine staining for IFN- $\gamma$ followed by flow cytometry analysis. As shown in Figure 4, tumorbearing mice that were administered MD5-1 mAb followed by CRT/E7(detox) DNA generated a significantly higher number of $\mathrm{E} 7$-specific $\mathrm{CD} 8^{+} \mathrm{T}$ cells compared to tumor-bearing mice that were administered CRT/E7(detox) DNA alone or MD5-1 mAb alone ( $p=0.001)$. These results indicate that treatment of tumor-bearing mice with MD5-1 mAb followed by CRT/E7 (detox) DNA leads to the strongest E7-specific CD8+ T cell immune response.

\section{Treatment with MD5-1 mAb renders the TC-1 tumor cells more susceptible to lysis by E7- specific CTLs}

In order to determine if treatment of TC-1 tumor cells with MD5-1 mAb will render the tumor cells more susceptible to E7-specific T cell-mediated killing, we performed a cytotoxicity assay using luciferase-expressing TC-1 tumor cells. TC-1 tumor cells were treated with MD5-1 mAb alone, MD5-1 mAb and E7-specific cytotoxic T cells (CTL), or treated with E7-specific CTLs alone. Untreated TC-1 tumor cells were used as a control. The CTL-mediated killing of the TC-1 tumor cells in each well was monitored using bioluminescent imaging systems. The 
degree of CTL-mediated killing of the tumor cells was indicated by the decrease of luminescence activity. As shown in Figure 5, the lowest luciferase activity was observed in the wells incubated with MD5-1 mAb and E7-specific CTLs as compared to the wells incubated with MD5-1 mAb alone or E7-specific CTLs alone $(p=0.001)$. Thus, our data suggests that the TC-1 tumor cells treated with MD5-1 mAb increased the susceptibility of the tumor cells for lysis by the E7-specific cytotoxic T cells.

\section{Discussion}

In the current study, we observed that E7-expressing TC-1 tumors expressed DR5. TC-1 tumorbearing mice treated with MD5-1 mAb followed by CRT/E7(detox) DNA were found to generate the highest frequency of E7-specific CD8+ T cells as well as potent therapeutic antitumor effects. We also found that treatment with MD5-1 mAb was able to render the TC-1 tumor cells more susceptible to lysis by E7-specific CTLs.

We observed that the combination of anti-DR5 mAbs with CRT/E7(detox) DNA vaccines could significantly enhance the E7-specific CD8+ T cell immune responses in vaccinated mice compared to CRT/E7(detox) DNA vaccination alone. The observed enhancement of E7specific CD8+ T cell immune response is most likely related to apoptotic tumor cell death caused by treatment with MD5-1 [13]. It has been shown that the binding of anti-DR5 antibodies to DR5 on tumor cells leads to apoptotic tumor cell death [21]. Furthermore, we have previously shown that apoptotic tumor cell death generated by chemotherapeutic agents such as EGCG [23] or cisplatin [24] are able to increase the frequency of E7-specific CD8+ T cell precursors. Thus, pretreatment with MD5-1 mAb would lead to apoptotic tumor cell death, and result in increased frequency of tumor antigen-specific $\mathrm{CD} 8+\mathrm{T}$ cell precursors. Thus, CRT/E7(detox) DNA vaccination following MD5-1 treatment would generate significantly enhanced E7-specific CD8+ T cell immune responses, resulting in potent antitumor effects.

We have chosen HPV-16 E7 as the target antigen for our therapeutic HPV DNA vaccine development. HPV is one of the most common sexually transmitted diseases in the world and persistent HPV infection with high-risk types such as HPV-16 have been considered as an etiological factor for cervical cancer [28]. HPV DNA has been detected in more than $99.7 \%$ of cervical cancers [29]. In particular, HPV-16 accounts for more than $50 \%$ of all cervical cancers (For review see [30]). The HPV early viral protein, E7 that is used in the current study, is a potentially ideal target antigen for the DNA vaccine development. E7 expression is identified in all HPV-associated cancer cells but not in normal cells and E7 is essential for malignant transformation of HPV-associated cancer cells. Thus, HPV-16 E7 is an ideal target antigen for DNA vaccine development and can potentially be used for the control of HPVassociated malignancies.

In summary, our study demonstrated that the combination of treatment with the anti-DR5 antibody and CRT/E7(detox) DNA vaccination generated significant E7-specific CD8+ T cell immune responses and resulted in potent therapeutic effects against TC-1 tumors in tumorbearing mice compared to either treatment alone. For eventual clinical translation, it would be important to further determine the optimal dose and regimen of the anti-DR5 antibody and CRT/E7(detox) DNA vaccine in order to generate the best therapeutic effects in a preclinical model.

\section{Acknowledgments}

We gratefully acknowledge Ms. Talia Hoory for assistance in preparation of the manuscript. This work was supported by the Flight Attendant Medical Research Institute and National Cancer Institute SPORE in Cervical Cancer P50 CA098252 and the 1 RO1 CA114425-01. 


\section{References}

1. Donnelly JJ, Ulmer JB, Liu MA. DNA vaccines. Life Sci 1997;60(3):163-72. [PubMed: 9000640]

2. Gurunathan S, Klinman DM, Seder RA. DNA vaccines: immunology, application, and optimization. Annu Rev Immunol 2000;18:927-74. [PubMed: 10837079]

3. Hung CF, Wu TC. Improving DNA vaccine potency via modification of professional antigen presenting cells. Curr Opin Mol Ther 2003 Feb;5(1):20-4. [PubMed: 12669466]

4. Tsen SW, Paik AH, Hung CF, Wu TC. Enhancing DNA vaccine potency by modifying the properties of antigen-presenting cells. Expert Rev Vaccines 2007 Apr;6(2):227-39. [PubMed: 17408372]

5. Rice J, Ottensmeier CH, Stevenson FK. DNA vaccines: precision tools for activating effective immunity against cancer. Nat Rev Cancer 2008 Feb;8(2):108-20. [PubMed: 18219306]

6. Gelebart P, Opas M, Michalak M. Calreticulin, a Ca2+-binding chaperone of the endoplasmic reticulum. Int J Biochem Cell Biol 2005 Feb;37(2):260-6. [PubMed: 15474971]

7. Cheng WF, Hung CF, Chai CY, Hsu KF, He L, Ling M, et al. Tumor-specific immunity and antiangiogenesis generated by a DNA vaccine encoding calreticulin linked to a tumor antigen. J Clin Invest 2001 Sep;108(5):669-78. [PubMed: 11544272]

8. Kim JW, Hung CF, Juang J, He L, Kim TW, Armstrong DK, et al. Comparison of HPV DNA vaccines employing intracellular targeting strategies. Gene Ther 2004 Jun;11(12):1011-8. [PubMed: 14985791]

9. Trimble C, Lin CT, Hung CF, Pai S, Juang J, He L, et al. Comparison of the CD8+ T cell responses and antitumor effects generated by DNA vaccine administered through gene gun, biojector, and syringe. Vaccine 2003 Sep 8;21(2526):4036-42. [PubMed: 12922140]

10. Munger K, Basile JR, Duensing S, Eichten A, Gonzalez SL, Grace M, et al. Biological activities and molecular targets of the human papillomavirus E7 oncoprotein. Oncogene 2001 Nov 26;20(54): 7888-98. [PubMed: 11753671]

11. Heck DV, Yee CL, Howley PM, Munger K. Efficiency of binding the retinoblastoma protein correlates with the transforming capacity of the E7 oncoproteins of the human papillomaviruses. Proc Natl Acad Sci U S A 1992 May 15;89(10):4442-6. [PubMed: 1316608]

12. Edmonds C, Vousden KH. A point mutational analysis of human papillomavirus type $16 \mathrm{E} 7$ protein. J Virol 1989 Jun;63(6):2650-6. [PubMed: 2542578]

13. Takeda K, Yamaguchi N, Akiba H, Kojima Y, Hayakawa Y, Tanner JE, et al. Induction of tumorspecific T cell immunity by anti-DR5 antibody therapy. J Exp Med 2004 Feb 16;199(4):437-48. [PubMed: 14769851]

14. Chaudhary PM, Eby M, Jasmin A, Bookwalter A, Murray J, Hood L. Death receptor 5, a new member of the TNFR family, and DR4 induce FADD-dependent apoptosis and activate the NF-kappaB pathway. Immunity 1997 Dec;7(6):821-30. [PubMed: 9430227]

15. Kendrick JE, Straughn JM Jr, Oliver PG, Wang W, Nan L, Grizzle WE, et al. Anti-tumor activity of the TRA-8 anti-DR5 antibody in combination with cisplatin in an ex vivo human cervical cancer model. Gynecol Oncol. 2008 Jan 3;

16. Takeda K, Stagg J, Yagita H, Okumura K, Smyth MJ. Targeting death-inducing receptors in cancer therapy. Oncogene 2007 May 28;26(25):3745-57. [PubMed: 17530027]

17. Walczak H, Haas TL. Biochemical analysis of the native TRAIL death-inducing signaling complex. Methods Mol Biol 2008;414:221-39. [PubMed: 18175822]

18. Straughn JM Jr, Oliver PG, Zhou T, Wang W, Alvarez RD, Grizzle WE, et al. Anti-tumor activity of TRA-8 anti-death receptor 5 (DR5) monoclonal antibody in combination with chemotherapy and radiation therapy in a cervical cancer model. Gynecol Oncol 2006 Apr;101(1):46-54. [PubMed: $16271751]$

19. Kendrick JE, Estes JM, Straughn JM Jr, Alvarez RD, Buchsbaum DJ. Tumor necrosis factor-related apoptosis-inducing ligand (TRAIL) and its therapeutic potential in breast and gynecologic cancers. Gynecol Oncol 2007 Sep;106(3):614-21. [PubMed: 17602728]

20. Estes JM, Oliver PG, Straughn JM Jr, Zhou T, Wang W, Grizzle WE, et al. Efficacy of anti-death receptor 5 (DR5) antibody (TRA-8) against primary human ovarian carcinoma using a novel ex vivo tissue slice model. Gynecol Oncol 2007 May;105(2):291-8. [PubMed: 17303227] 
21. McKee CM, Ye Y, Richburg JH. Testicular germ cell sensitivity to TRAIL-induced apoptosis is dependent upon $\mathrm{p} 53$ expression and is synergistically enhanced by DR5 agonistic antibody treatment. Apoptosis 2006 Dec;11(12):2237-50. [PubMed: 17051329]

22. Plummer R, Attard G, Pacey S, Li L, Razak A, Perrett R, et al. Phase 1 and pharmacokinetic study of lexatumumab in patients with advanced cancers. Clin Cancer Res 2007 Oct 15;13(20):6187-94. [PubMed: 17947486]

23. Kang TH, Lee JH, Song CK, Han HD, Shin BC, Pai SI, et al. Epigallocatechin-3-gallate enhances CD8+ T cell-mediated antitumor immunity induced by DNA vaccination. Cancer Res 2007 Jan 15;67 (2):802-11. [PubMed: 17234792]

24. Tseng CW, Hung CF, Alvarez RD, Trimble C, Huh WK, Kim D, et al. Pretreatment with Cisplatin Enhances E7-Specific CD8+ T-Cell-Mediated Antitumor Immunity Induced by DNA Vaccination. Clin Cancer Res 2008 May 15;14(10):3185-92. [PubMed: 18483387]

25. Lin KY, Guarnieri FG, Staveley-O'Carroll KF, Levitsky HI, August JT, Pardoll DM, et al. Treatment of established tumors with a novel vaccine that enhances major histocompatibility class II presentation of tumor antigen. Cancer Res 1996 Jan 1;56(1):21-6. [PubMed: 8548765]

26. Kayagaki N, Yamaguchi N, Nakayama M, Takeda K, Akiba H, Tsutsui H, et al. Expression and function of TNF-related apoptosis-inducing ligand on murine activated NK cells. J Immunol 1999 Aug 15;163(4):1906-13. [PubMed: 10438925]

27. Chen CH, Wang TL, Hung CF, Yang Y, Young RA, Pardoll DM, et al. Enhancement of DNA vaccine potency by linkage of antigen gene to an HSP70 gene. Cancer Res 2000 Feb 15;60(4):1035-42. [PubMed: 10706121]

28. Walboomers JM, Jacobs MV, Manos MM, Bosch FX, Kummer JA, Shah KV, et al. Human papillomavirus is a necessary cause of invasive cervical cancer worldwide. J Pathol 1999;189(1):129. [PubMed: 10451482]

29. Bosch FX, Munoz N, de Sanjose S. Human papillomavirus and other risk factors for cervical cancer. Biomed Pharmacother 1997;51(67):268-75. [PubMed: 9309247]

30. zur Hausen H. Papillomaviruses and cancer: from basic studies to clinical application. Nat Rev Cancer 2002 May;2(5):342-50. [PubMed: 12044010] 


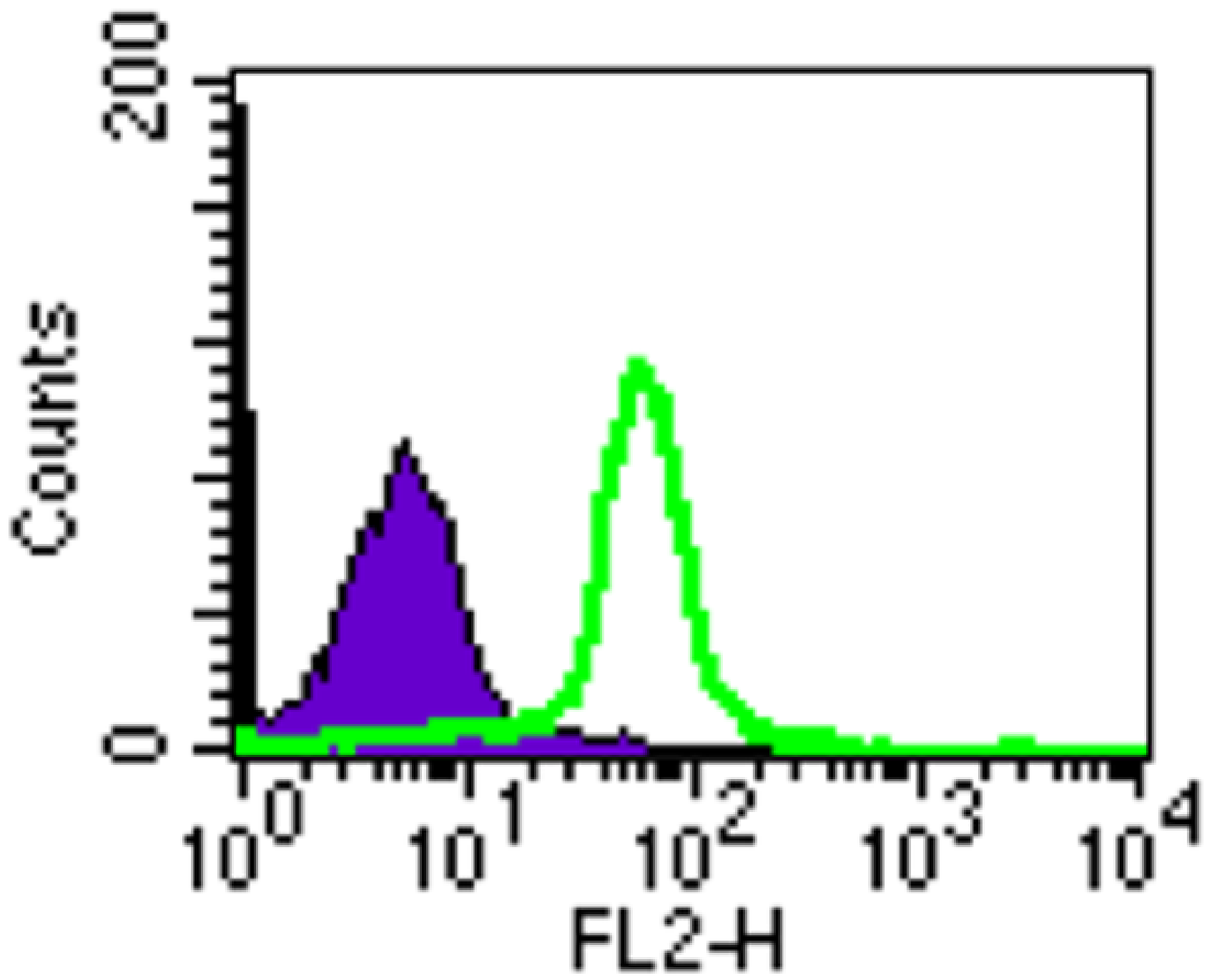

Figure 1. Flow cytometry analysis to demonstrate the expression of DR5 on TC-1 tumor cells TC-1 cells were isolated and stained with PE-conjugated MD5-1 mAb to detect the expression of DR5. Flow cytometry analysis was performed to characterize the expression of DR5 on the TC-1 tumor cells. The green profile represents the TC-1 tumor cells. The isotype-matched control antibody was used as the negative control (purple profile). 
(A)

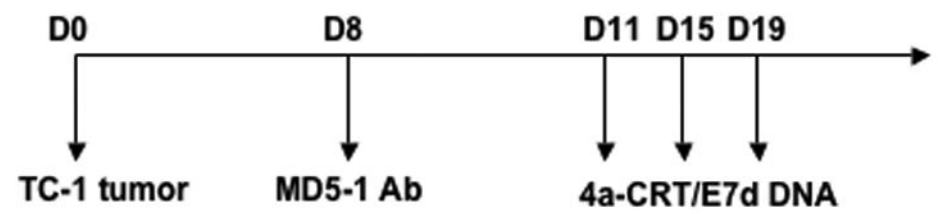

(B)

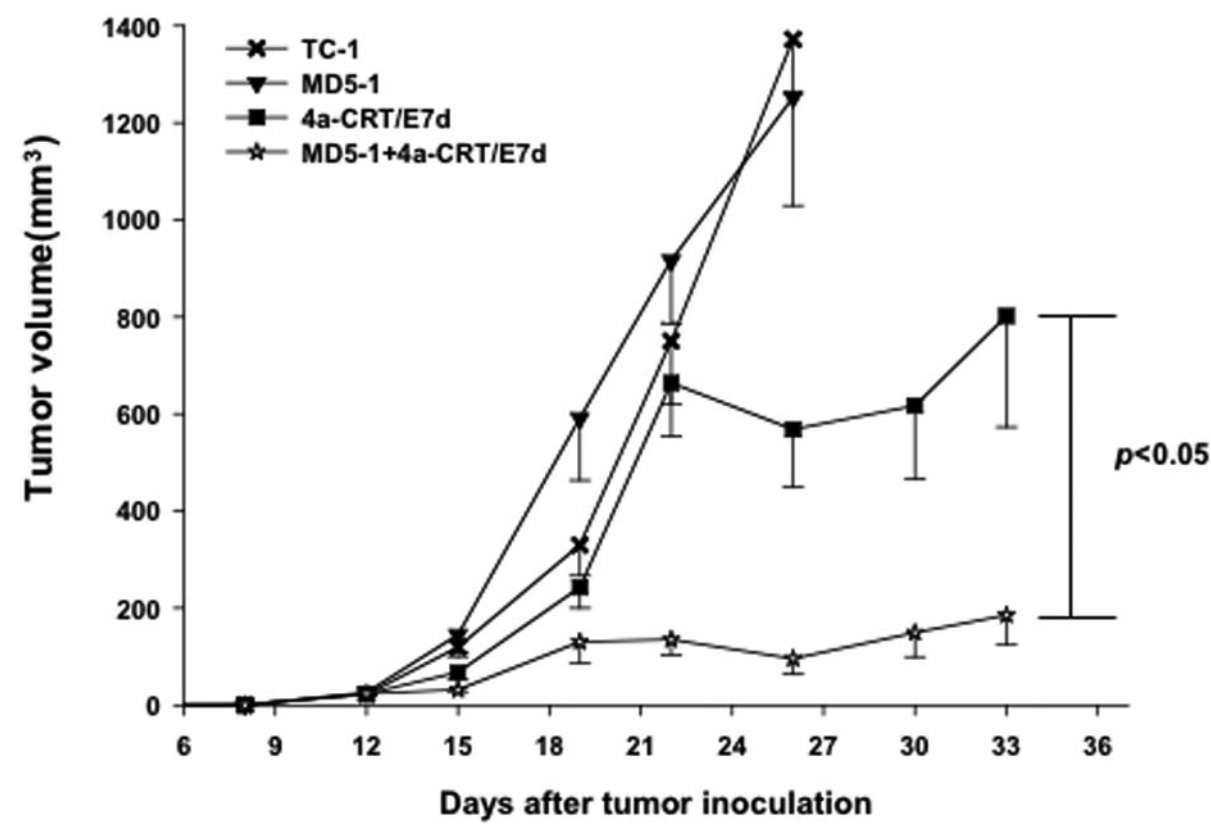

Figure 2. In vivo treatment experiment in tumor-bearing mice treated with MD5-1 mAb and/or CRT/E7(detox) DNA vaccine

(A) Schematic diagram showing the treatment regimen of MD5-1 $\mathrm{mAb}$ and/or the CRT/E7 (detox) DNA vaccine. (B) Line graph depicting the tumor volume in TC-1 tumor-bearing mice treated with MD5-1 mAb and/or CRT/E7(detox) DNA vaccine. Groups of C57BL/6 mice (5 per group) were subcutaneously challenged with $5 \times 10^{4} /$ mouse of TC- 1 tumor cells on day 0 . Tumor-bearing mice were treated with $0.25 \mathrm{mg} / \mathrm{mouse}$ of MD5-1 mAb intraperitoneally on day 8. Three days later, the mice were vaccinated with or without DNA encoding CRT/E7 (detox) DNA via gene gun in the amount of $2 \mathrm{ug} /$ mouse three times with 4-day intervals. A group of mice treated with the DNA vaccine alone were used as a control. Data are represented as mean \pm s.e. Data shown are representative of two experiments performed. 


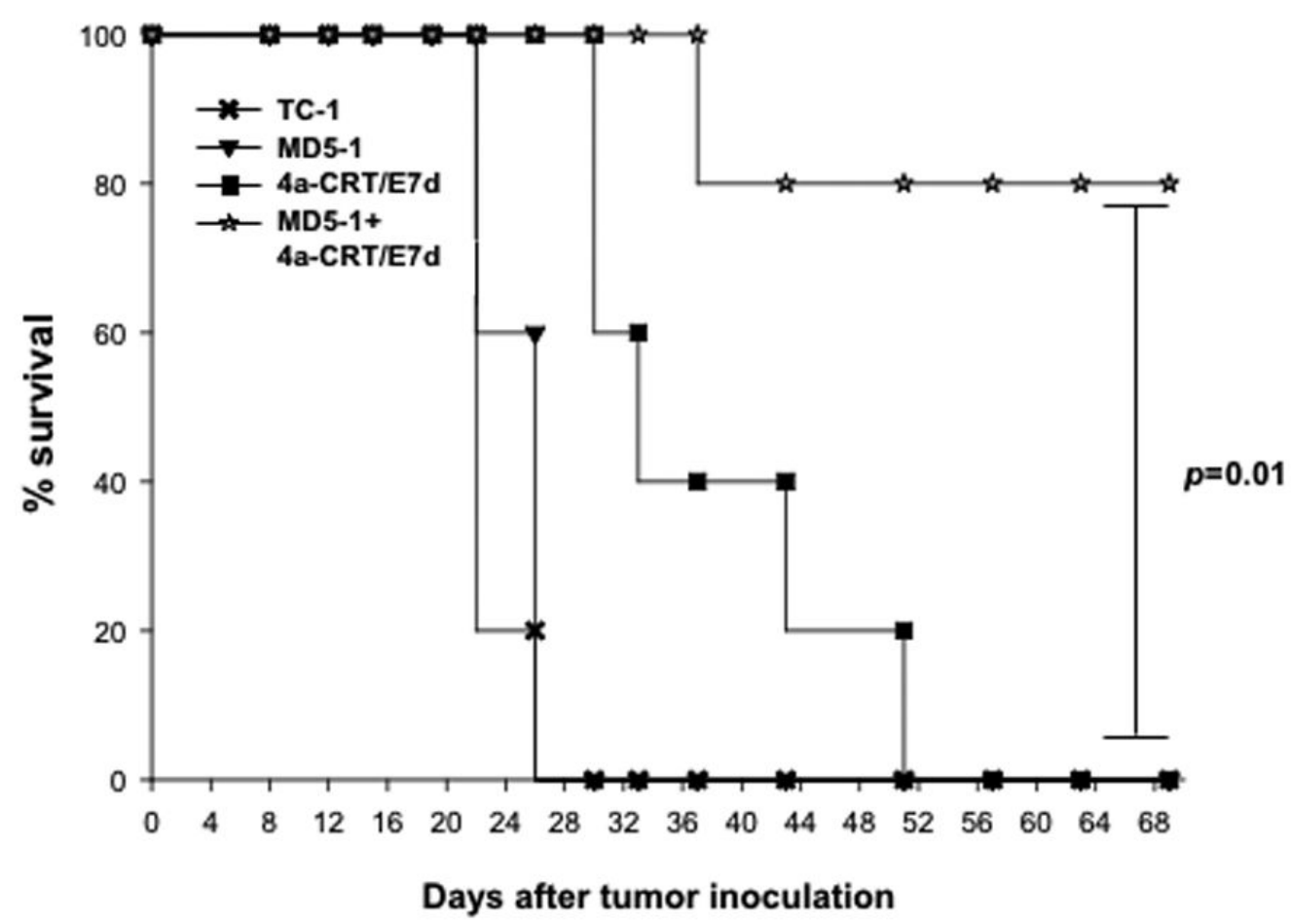

Figure 3. Kaplan Meier survival analysis in tumor-bearing mice treated with MD5-1 mAb and/or CRT/E7(detox) DNA vaccine

Groups of C57BL/6 mice (5 per group) were challenged with TC-1 tumor cells and treated with MD5-1 mAb and/or vaccinated with CRT/E7(detox) DNA as described in Figure 2A. Kaplan \& Meier survival analysis of TC-1 tumor-bearing mice treated with MD5-1 mAb and/ or CRT/E7(detox) DNA vaccine. 


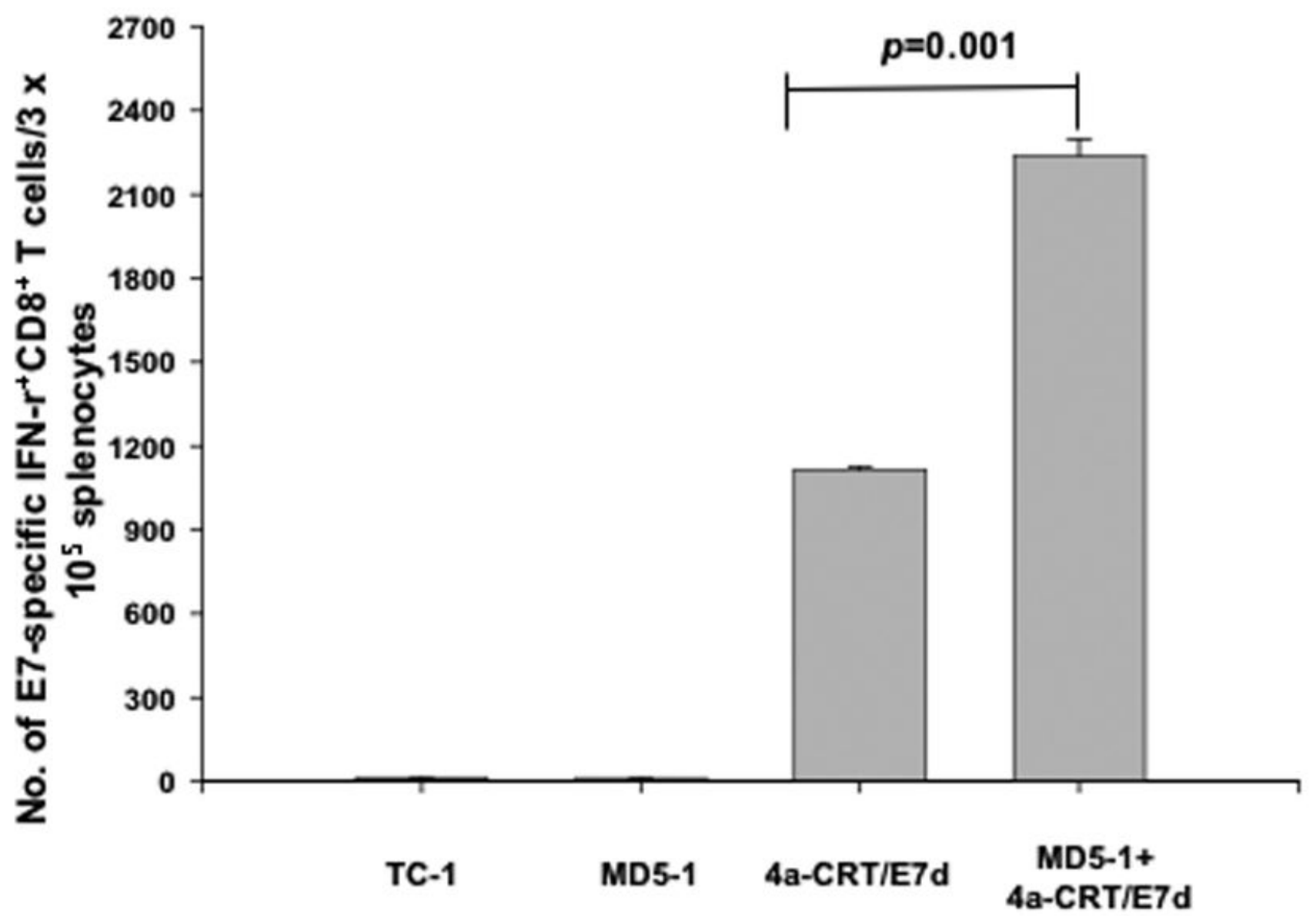

Figure 4. Intracellular cytokine staining followed by flow cytometry analysis to determine the number of E7-specific $\mathrm{CD8}^{+} \mathrm{T}$ cells in tumor-bearing mice treated with MD5-1 and/or CRT/E7 (detox) DNA

Groups of C57BL/6 mice (5 per group) were challenged with TC-1 tumor cells and treated with MD5-1 and/or CRT/E7(detox) DNA as illustrated in Figure 2A. One week after the last vaccination, splenocytes from tumor-bearing mice were harvested and characterized for E7specific $\mathrm{CD}^{+} \mathrm{T}$ cells using intracellular IFN- $\gamma$ staining followed by flow cytometry analysis. Data is represented as bar graph depicting the numbers of E7-specific IFN- $\gamma$-secreting CD8 ${ }^{+}$ $\mathrm{T}$ cells per $3 \times 10^{5}$ pooled splenocytes (mean \pm s.e.). Data shown are representative of two experiments performed. 
(A)

(B)
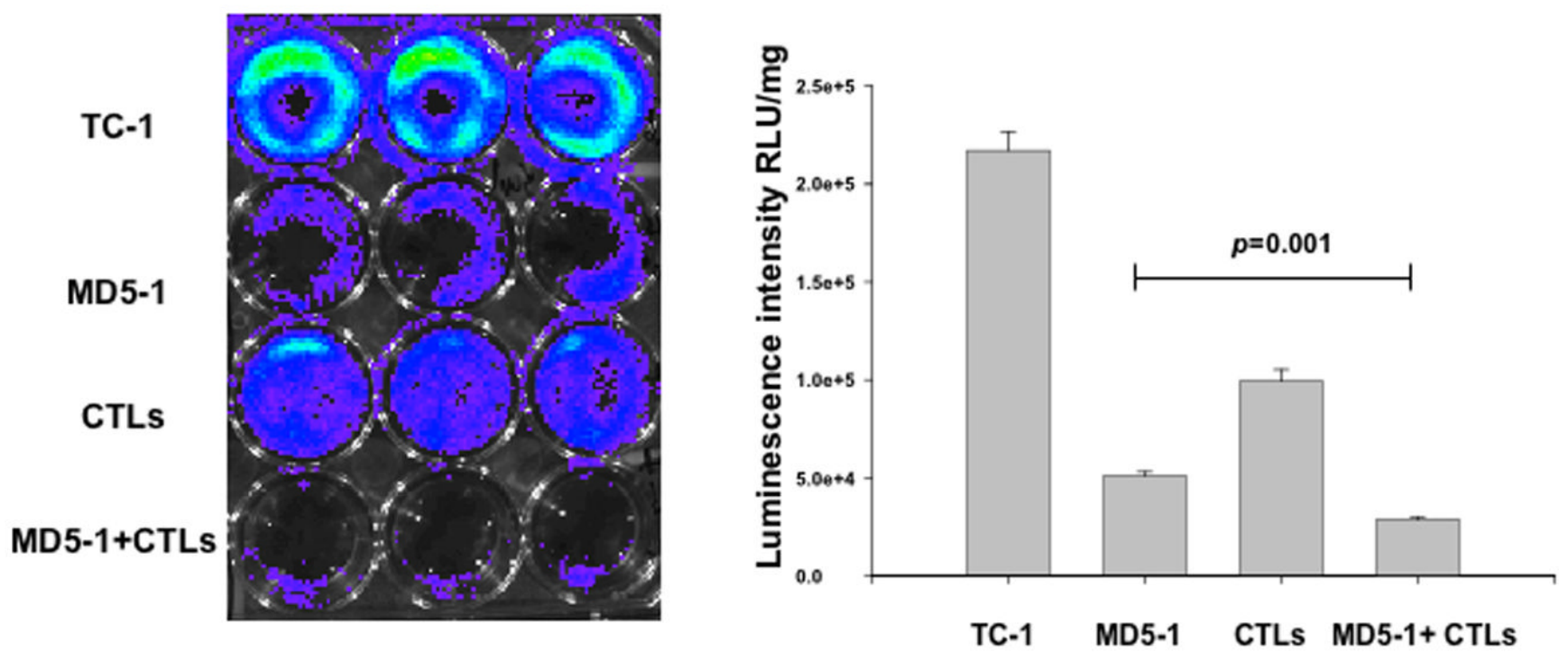

Figure 5. In vitro cytotoxicity assay

Luciferase-expressing TC- 1 tumor cells were added to 24 -well plates at a dose of $1 \times 10^{5} /$ well. Three days later, TC- 1 tumor cells were treated with either $5 \mu$ l of MD5-1 mAb $(2.5 \mathrm{mg} / \mathrm{ml})$ alone, $11 \times 10^{5}$ E7-specific cytotoxic T cells (CTL) alone, co-treated with MD5-1 mAb and $11 \times 10^{5}$ E7-specific cytotoxic T cells (CTL), or left untreated. The degree of CTL-mediated killing of the tumor cells was indicated by the decrease of luminescence activity using the IVIS luminescence imaging system series 200. Bioluminescence signals were acquired for one minute. A) Representative luminescence images of 24-well plates showing lysis of the tumor cells. B) Bar graph depicting the quantification of luminescence intensity in tumor cells treated with MD5-1 mAb and/or E7-specific cytotoxic T cells (mean \pm s.e.). 